\title{
Two-dimensional optical coherence tomography for real- time structural dynamical characterization
}

\author{
Jianfeng Zhong, ${ }^{1}$ Shuncong Zhong, ${ }^{1,2, *}$, Qiukun Zhang ${ }^{1}$ and Ligang Yao' \\ ${ }^{1}$ Laboratory of Optics, Terahertz and Non-destructive Testing, School of Mechanical \\ Engineering and Automation, Fuzhou University, Fuzhou 350108, P. R. China \\ ${ }^{2}$ Fujian Key Laboratory of Medical Instrument and Pharmaceutical Technology, Fuzhou \\ 350108, P.R. China \\ Correspondence: sczhong@fzu.edu.cn
}

\begin{abstract}
We present a two-dimensional optical coherence vibration tomography (2DOCVT) system with an ultra-precision displacement resolution of $\sim 0.1 \mathrm{~nm}$ that is capable of in site realtime absolute displacement measurement of structural line vibrations. Experimental results of sinusoidal, sweep and impulse vibrations were reported. The key figures of merit such as the 2DOCVT system could obtain fast line vibration measurement without scanning and it also could be used to capture structural modal parameters in one single impulse excitation measurement without any vibration excitation input information, making it attractive for the application in low-frequency vibration measurement and response-only modal analysis.
\end{abstract}

Key words: Two-dimensional Optical Coherence Tomography, absolute displacement measurement

\section{Introduction}

Conventional Laser Doppler vibrometry [1-4] are widely used in mechanical vibration measurement. These methods exhibit high reliability and enable wideband, phase-resolved, single-point measurements. We previously reported a single-point spectral-domain optical coherence vibration tomography (OCVT) [5] to quantify both vibration the inner structure (layer thickness) of a vibrating sample. However, these single-point methods require timeconsuming scanning of the tested sample [6]. Recently, Fu et al. [7-9] have employed single photodetector or high-speed imaging for multi-point laser Doppler vibrometry which allows 20-point measurement. Haist et al. [10] used a spatial light modulator-based multipoint vibrometer realizing 14 point independent fast heterodyne interferometry channels for measuring vibrations. Waz et al. [11] built a multi-point fibre laser vibrometry based on twochannel wavelength division multiplexing (WDM) technique in fibre telecommunications. Two-point vibration measurement by a 2-point fiber laser vibrometry was demonstrated. MacPherson et al. [12] reported a multipoint laser vibrometer which is capable of resolving 256 measurment points. Speckle interferometry [13-16], holographic interferometry $[6,17,18]$, heterodyne interferometer [19] and Self-mixing interference [20] enable reliable measurements of mechanical vibrations. However, for these interferometer techniques [1-4, 6-20], the displacement is obtained from the phase change of the interferometric patterns, providing extremely high depth resolution (down to $10^{-12} \mathrm{~m}$ or $1 \mathrm{pm}$ ) [21]. However, the detection range is usually limited to half the wavelength of the laser source owing to the $2 \pi$ phase ambiguity [2]. The detection range can be extended beyond the half wavelength limit by using phase shift 
modulation (or phase unwrapping) techniques, at the cost of increased instrument complexity and decreased measurement accuracy (related to the inherent phase shift error) [14]. On the other hand, laser Doppler vibrometre directly measures the velocity of a sample although the displacement information is an analog integration of the velocity signal along the time axis [2223]. However, such analog integrators always require a high pass filtering of the velocity signal. Thus DC measurements are not possible, but are limited in their dynamical range and additional errors are added to the calibration chain of the system [23]. For digital decoder, laser Doppler vibrometre can retrieve displacement by directly extracting the phase [23]. We report a twodimensional Fourier-domain optical coherence vibration tomography (2DOCVT) system and experimental demonstration of absolute displacement measurement of line vibrations in real time by directly measuring the displacement of structures using the home-made system. Different from the phase-sensitive OCT, the digital-decoding laser Doppler vibrometre and other interferometer techniques, the 2DOCVT system is based on the accurate amplitude not on the unwrapped phase value of the FFT of spectral interferogram. Additionally, the 2DOCVT could also work as a traditional OCT which could quantify inner structure information of static samples, like OCVT. The distinguished feature of the 2DOCVT system is that it could measure a series number of points in the vibrating structure simultaneously without scanning which is the major difference with the OCVT system in which point-by-point scanning will be necessary if one wants to obtain a line vibration.

\section{Two-dimensional Optical Coherence Vibration Tomography}

A schematic diagram of a 2DOCVT for the measurement of line vibration is shown in Fig.1. The light from a high-intensity tungsten halogen lamp (OSL1-EC, Thorlabs) was focused on a multimode optical fiber using a biconvex lens and delivered onto another biconvex lens. A line focus is created using a cylindrical lens and it was then imaged at a ratio of 1:1 onto the surface of a reference and the vibrating sample using a beam-splitter (50:50). The line light that was reflected or scattered back from both the reference and the sample was collimated using another cylindrical lens and imaged back onto a reference and then to a reflection grating and an interferometer. Interference will occur when both the "sample image" and "reference image" are spatially matched in size and orientation within the corresponding transverse coherence distance, and their path lengths are matched within the coherence length of the light source. A holographic reflective grating $(600$ lines $/ \mathrm{mm})$ placed in the Fourier plane of the imaging system and the line light was captured by a high-speed CCD camera (PCO.1200hs, PCO-TECH, Germany). In this way, interference fringe of each point from different position of line focus is imaged on the corresponding line pixels of the CCD camera, and a full spectral interferogram was recorded for all points of the line focus simultaneously. The CCD camera contains $1280 \times 1024$ pixels. The exposure time was set to 0.000789 s. We set the resolution to $1280 \times 512$ pixels, so 512 A-scans were recorded in parallel for each camera exposure. Only 465 pixels (from No. 31 to 495 pixel) of CCD camera were used in the analysis because the line focus only covered 465 CCD pixels in width. The sample was attached to a loudspeaker that acted as a vibration exciter. The vibration amplitude and frequency of the sample were controlled by the signal voltage and frequency from a signal generator (Agilent 33220). 
In the Fourier-domain optical coherence vibration tomography, a spectral interferogram is recorded from a reference electric field $E r$ and an electric field Es reflected/scattered from the surface of the sample. The spectral interferogram $I(\lambda, y, t)$ can be expressed as

$$
I(\lambda, y, t)=\left|E_{r}(\lambda, y, t)\right|^{2}+\left|E_{s}(\lambda, y, t)\right|^{2}+2\left|\mathrm{E}_{\mathrm{r}}(\lambda, \mathrm{y}, \mathrm{t}) \mathrm{E}_{\mathrm{s}}(\lambda, \mathrm{y}, \mathrm{t})\right| \cos (\Delta \phi(\lambda, \mathrm{y}, \mathrm{t}))
$$

where $\lambda$ is the wavelength of the source light, $y$ is the coordinate of the line focus on the vibrating sample, $\mathrm{t}$ is time, and $\Delta \phi(\lambda, \mathrm{y}, \mathrm{t})$ is a phase term resulting from the optical path length difference $\Delta \mathrm{Z}(\mathrm{y}, \mathrm{t})$ between the reference and the sample arm as

$$
\Delta \phi(\lambda, \mathrm{y}, \mathrm{t})=\phi_{\mathrm{r}}(\lambda, \mathrm{y}, \mathrm{t})-\phi_{\mathrm{s}}(\lambda, \mathrm{y}, \mathrm{t})=4 \pi \mathrm{n} \Delta \mathrm{Z}(\mathrm{y}, \mathrm{t}) / \lambda
$$

The Fast Fourier Transform (FFT) of each measured spectral interferogram provides us the surface position (i.e. $\Delta \mathrm{Z}(\mathrm{y}, \mathrm{t})$ ) which is a function of $\mathrm{t}$ in $\mathrm{y}$ coordinates in line. For a static sample, that is, when time $t$ in Eq. (1) is a constant, the system could be worked as a traditional optical coherence tomography [24-26]. For a single-point vibration measurement using a spectroscopy, such as OCVT system [5], the coordinate of the line focus y in Eq. (1) is a constant as well. That means that if the OCVT system wants to measure a line vibration of a structure, the time-consuming scanning will be necessary. Furthermore, the changing environment will affect the vibration acquisition during the point-by-point measurement. For our 2DOCVT system, the line vibration could be obtained in one single measurement which means all the point vibrations of the line were acquired simultaneously.

Due to signal leakage effects [27], the amplitude, phase and frequency calculated from the FFT of the measured spectral interferogram are normally different from the real one even in the absence of noise. Applying windowing functions to the measured spectral interferogram is a common method to minimize the effect of leakage. However, theoretically, the maximum relative error of the amplitude estimation will be $36.4 \%$ and $15.3 \%$ respectively, when Rectangle and Hanning windows are employed to minimize leakage [28]. When Hanning window is used in the FFT of a measured spectral interferogram, a spectral center correction method (SCCM) [27] could be used to obtain almost exact determination of signal parameters such as frequency and amplitude, etc.

Simulated spectral interferogram of 2DOCVT system were computed using Eq. (1), in which $\Delta \mathrm{Z}(\mathrm{y}, \mathrm{t})$ is a $5 \mathrm{~Hz}$ sine wave with displacement amplitudes of $700 \mathrm{~nm}$ and $1 \mathrm{~nm}$ respectively. For $700 \mathrm{~nm}$ vibration, the surface displacement of the vibrating sample (i.e., the main peak of OCT-FFT waveform) could not be accurately calculated due to the inherent shortcoming of FFT (leakage effect), as the dashed line in Fig.2 (a). If the FFT of the simulated spectral interferogram were corrected by SCCM, the sine vibration with $700 \mathrm{~nm}$ could be obtained precisely, as the solid line in Fig.2 (a). The line vibration calculated using the simulated 2DOCVT spectral interferogram for $700 \mathrm{~nm}$ vibration was demonstrated in Fig.2 (b). Similarly, for $1 \mathrm{~nm}$ sine vibration, no displacement (dashed line) was obtained without SCCM, as shown in Fig.2(c). It meant that without SCCM, the 2DOCVT has no capability in measurement of nanoscale vibration which is beyond axial resolution of the system. However, if the FFT of the simulated spectral interferograms were corrected by SCCM, the very small vibration displacement with amplitude of $1 \mathrm{~nm}$ could be obtained, as shown in the solid line in 
Fig.2 (c). Fig.2 (d) is the corresponding line vibration for $1 \mathrm{~nm}$ sine vibration. From the simulation results, the displacement resolution for the developed 2DOCVT system, which is better than $0.1 \mathrm{~nm}$, could be achieved.

\section{Experiments and discussions}

Fig.3 (a) showed the interferogram of "sample image" and "reference image" in the cylindrical line focus for a static sample. The overall length of line focus was $8.92 \mathrm{~mm}$ which covered 465 CCD pixels in width; therefore, the longitudinal resolution was $19.18 \mu \mathrm{m}$. Fig.3 (b) was the corresponding FFT of the interferogram shown in Fig.3 (a). The line located at the position of $35.2 \mu \mathrm{m}$ indicated the surface position of the sample and it means that there was an optical path distance of $35.2 \mu \mathrm{m}$ between the sample and the reference. Fig.3 (c) showed the OCT spectral interferogram from No. 400 pixel of the CCD camera and its corresponding FFT result showed the surface position of the sample at $35.2 \mu \mathrm{m}$. In the meanwhile, it can be seen from Fig.3 (b), the line at $35.2 \mu \mathrm{m}$ was almost a straight line which indicated that the sample has a flat surface. Therefore, the developed system had the capability in surface characterization and assessment.

The measured spectrum had a center wavelength of $\lambda_{0}=705 \mathrm{~nm}$ and a full width at halfmaximum (FWHM) of $\Delta \lambda=136 \mathrm{~nm}$. The axial resolution of the 2DOCVT system for internal imaging was calculated to be $1.163 \mu \mathrm{m}$ in air, using the expression of $2 \ln 2 \lambda_{0}{ }^{2} / \pi \Delta \lambda$ [25]. However, as shown previously, the FFT results of interferogram were corrected by SCCM to obtain high accuracy vibration amplitude in nanoscale. To achieve the displacement resolution of the developed 2DOCVT, a $10 \mathrm{~Hz}$ sine wave with amplitudes of $10 \mathrm{mv}, 20 \mathrm{mv}, 50 \mathrm{mv}$ and $200 \mathrm{mv}$ voltages were used to drive a multilayer piezoelectric stack actuator (Thorlabs). This caused the surface of the PZT actuator to vibrate at a frequency of $10 \mathrm{~Hz}$ with amplitudes in nanoscale. The 2DOCVT system was installed on an active vibration isolation optical table in a quiet room with constant temperature and humidity. In order to separate the vibration excited by such as environment noise, a low-pass filter was applied to the measured vibration signals. As shown in Fig.4 (a), the dashed line is the low-pass filtered signal which includes the vibration excited by PZT actuator by $10 \mathrm{~Hz}$ sine wave with amplitude of $20 \mathrm{mv}$ voltage, and also the lowerfrequency noise. To further extract the vibration excited only by PZT actuator, a narrow bandpass filter was employed to pick up the $10 \mathrm{~Hz}$ sine vibration. The band-pass filtered vibration signal is shown as the solid line in Fig.4 (a), from which the vibration amplitude of $1.72 \mathrm{~nm}$ was obtained. Using the similar signal filtering strategy, the vibrations excited by PZT actuator by $10 \mathrm{~Hz}$ sine wave with amplitudes of $10 \mathrm{mv}, 40 \mathrm{mv}$ and $200 \mathrm{mv}$ voltages were measured, as shown in Fig.4 (b). The corresponding amplitudes for $10 \mathrm{mv}$, $40 \mathrm{mv}$ and $200 \mathrm{mv}$ voltages are respectively $0.86,4.4$ and $17.3 \mathrm{~nm}$. It demonstrated excellent linearity of the PZT actuator in the range of nano-scale displacements. Therefore, such very nanoscale displacements could be accurately characterized by the developed 2DOCVT system whose displacement resolution is about $0.1 \mathrm{~nm}$.

For a vibrating sample, we could record the sequence of interferogram and therefore the line vibration signal of the sample in time domain is accessible. Fig. 5 showed the result of the vibration sample at a single-frequency measured by the developed 2DOCVT. Two frequencies of $5 \mathrm{~Hz}$ and $15 \mathrm{~Hz}$ sine signal from signal generator were respectively used to excite the loudspeaker. The $\pm 1 \mathrm{~V}$ voltage resulted in the vibration sample with the amplitude of $6.6 \mu \mathrm{m}$. For the two cases, the sampling frequency of the CCD was set to $1267 \mathrm{~Hz}$ (which was enough for real-time low-frequency vibration monitoring), and the CCD had a $1280 \times 512$ pixels resolution. Two three-dimensional waveforms in Fig.5 (a) showed the vibration of the sample 
excited by $5 \mathrm{~Hz}$ and $15 \mathrm{~Hz}$ sine signal. From Fig.5, we could find that the amplitudes of the two vibrations were $6.6 \mu \mathrm{m}$. The reason is that the amplitudes of the input voltage added to the loudspeaker were the same. It demonstrated the high precision and stability of the developed 2DOCVT system. In vibration engineering, a sweep source is generally used to excite a structure to get vibration parameters. The developed 2DOCVT system had the capability to deal with the vibrating sample with a sweep source. Fig.5 (b) showed the line vibration of the vibrating sample who was excited by a $0-20 \mathrm{~Hz}$ sweep source and whose vibration amplitude was $6.6 \mu \mathrm{m}$. It was demonstrated that this system could distinguish the vibration clearly in the line focus and each signal from adjacent points did not interfere with each other.

From the above experimental results, we could find that the distinguished feature of the 2DOCVT system is that it could measure a series number of points in the vibrating structure simultaneously, that is, it could obtain fast line vibration measurement without scanning. This is the major difference with the OCVT system in which point-by-point scanning will be necessary if one wants to obtain a line vibration. In the meanwhile, the 2DOCVT was to measure the absolute displacement of vibrating structures.

Normally, impulse excitation is used to characterize the vibration modal parameters of structures. We used the developed 2DOCVT system to monitor the vibration of a flat surface cantilevered beam which was excited by a shock impulse. The shock impulse was generated by a small metal bar (working like an impulse force hammer in traditional experimental modal analysis) which beat slightly near the fixed end of the cantilever beam to produce a very small vibration. In the experiment, we set the exposure time to $5 \mathrm{~ms}$ and the resolution to $1280 \times 300$ pixels for a longer data sampling due to the limit of memory. The line vibration could be recorded from the 2DOCVT line focus on the cantilevered beam, as shown in Fig.6 (a). The total acquisition time was 46.9s. As can be seen from Fig.6 (a), the vibration amplitudes of different positions at the cantilevered beam were different therefore the vibration modal shape could be obtained. To do a standard modal experimental analysis in current vibration engineering, the input force signal and the accelerometer output signal are needed to be acquired for analysis [27]. However, for our 2DOCVT system, it could obtain structural modal shape in one single impulse excitation measurement without any vibration excitation input information. Fig.6 (b) was the zoomed view for the line vibration at the range of 0 to 0.8 s. For clarity purposes, the point vibration signal from No. 270 pixel of CCD camera (approximately at the position of $5.18 \mathrm{~mm}$ ) was showed in Fig.6 (c). It can be seen from the figures, the vibration of the cantilevered beam was an attenuation signal due to the damping of the beam. Fig.6 (d) showed the result of frequency analysis of the experimental data in Fig.6 (a). As shown in Fig.6(d), all vibration amplitude information could be captured in a single impulse excitation experiment, it provides a novel tool to do modal shape analysis without any vibration excitation input information. Due to that the range of frequency measurement depends on the imaging rate of the camera, the sampling frequency of our current 2DOCVT system with a PCO-TECH high-speed CCD camera could be up to about $200 \mathrm{~Hz}$ for reliable measurement of both nanoand micro- scale vibrations. Therefore, our current developed 2DOCVT system is only suitable to measure the low-frequency vibration measurement and response-only modal analysis. However, the sampling frequency is dependent on the exposure time. Theoretically, shorter 
exposure time could be used for better surface reflection of the vibrating samples and therefore the sampling frequency could be increased correspondingly. In addition, if low resolution of the high-speed CCD camera with $86 \mathrm{MHz}$ pixelclock, for example $1280 \times 128$ pixels, is used in the image acquisition, the sampling frequency could be about $\sim 5 \mathrm{~K} \mathrm{~Hz}$ for the 2DOCVT system. Furthermore, the highest sampling frequency of $\sim 40 \mathrm{~K}$ for the $2 \mathrm{DOCVT}$ could be achieved if the lowest resolution of the PCO-TECH high-speed CCD camera was set. Therefore, the 2DOCVT has great potential for higher frequency applications, such as modal analysis of thin films, thin structures and micro-structures. This would be one of our future work.

\section{Concluding Remarks}

In conclusion, we present a Fourier-domain optical coherence vibration tomography (2DOCVT) system that is capable of absolute measuring the line vibration of the structure. The measurement results demonstrate the home-made system has high precision with an ultraprecision displacement resolution of $\sim 0.1 \mathrm{~nm}$ and excellent stability. In addition, the system is non-contact and noninvasive in nature. Therefore, 2DOCVT system might become a valuable and functional tool for several engineering applications such as high-precision manufacturing sector. The key figures of merit such as the 2DOCVT system could obtain fast line vibration measurement without scanning and it also could be used to capture structural modal shape in one single impulse excitation measurement without any vibration excitation input information, making it attractive for the application in low-frequency vibration measurement and responseonly structural modal analysis.

\section{Acknowledgement}

We gratefully acknowledge support from National Natural Science Foundation of China, Natural Science Foundation of Fujian Province, Training Program of Fujian Excellent Talents in University, Specialized Research Fund for the Doctoral Program of Higher Education, Ministry of Education, P.R. China (20133514110008) and Ministry of Health, P.R. China (WKJ-FJ-27).

\section{Reference}

1. A.B. Stanbridge, D.J. Ewins, "Modal Testing Using a Scanning Laser Doppler Vibrometer," Mech. Sys. Sig.1 Proc. 13(2), 255-270 (1999).

2. R. Dauksevicius R, A. bubulis, V. Jurenas, V. Ostasevicius, S. Tamulevicius, "Investigation of dynamics of cantilever-type microstructure by laser Doppler vibrometry," Ultragarsas 3, 29-32(2004).

3. T. Miles, M. Lucas, S. Rothberg, "Bending vibration measurement on rotors by laser vibrometry," Opt. Letters 21(4), 296-298 (1996).

4. C. Barriere and D. Royer, "Optical measurement of large transient mechanical displacements," Appl. Phy. Letter 79: 878(2001).

5. S. Zhong, H. Shen Y. C. Shen, "Real-time monitoring of structural vibration using spectraldomain optical coherence tomography," Opti. Laser Eng 49, 49: 127(2011).

6. N. Verrier and M. Atlan, "Absolute measurement of small-amplitude vibrations by timeaveraged heterodyne holography with a dual local oscillator," Opti. Letter 38(5), 739(2013).7. 
Y. Fu, M. Guo and P. B. Phua, "Spatially encoded multibeam laser Doppler vibrometry using a single photodetector", Opt. Lett. 35, 1356-1358 (2010)

8. Y. Fu, M. Guo, P. B. Phua, "Multi-point laser Doppler vibrometry with single detector: Principles, implementations and signal analyses," Applied Optics,50(10), 1280-1288 (2011).

9. Y. Fu, G. Pedrini, X. Li, "Interferometric Dynamic Measurement: Techniques Based on HighSpeed Imaging or a Single Photodetector", The Scientific World Journal, Vol.2014, 232906, http://dx.doi.org/10.1155/2014/232906 (2014)

10. T. Haist, C. Lingel, W. Osten, C. Rembe, M. Winter, and M. Giesen, "SLM-based multipoint vibrometry", Proc. SPIE 8082, Optical Measurement Systems for Industrial Inspection VII, 80821V; doi:10.1117/12.889337 (2011)

11. A T Waz, P R Kaczmarek and K M Abramski, "Laser-fibre vibrometry at 1550 nm”, 2009 Meas. Sci. Technol. 20, 105301, (2009)

12, W. N. MacPherson, M. Reeves, D. P. Towers, A. J. Moore, J. D. C. Jones, M. Dale and C. Edwards, "Multipoint laser vibrometer for modal analysis", Applied Optics, 46 (16), 3126-3132 (2007).

13. X. Li, G. Tao. "Low-frequency harmonic vibration analysis with temporal speckle pattern interferometry," Opti. Laser Tech. 34, 259-64(2002).

14. B. Dhanaseka, B. Ramamoorthy, "Digital speckle interferometry for assessment of surface roughness". Optics and Lasers in Engineering 2008; 46:272-80.

15. H. Lopes, F. Ferreira, J. V. Araújo dos Santos, P. Mereno-Gracia, "Localization of damage with speckle shearography and higher order spatial derivatives", Mech. Sys. Sig. Proc. (in press), 2014.

16. H. Lopes, J. Ribeiro, J.V. Araújo dos Santos, "Interferometric techniques in structural damage identification," Shock Vib. 19, 835-844( 2012).

17. P. Picart, J. Leval, J. C. Pascal, "2D full field vibration analysis with multiplexed digital holograms", Opt. Express 13(22), 8882-8892( 2005).

18. Picart, E. Moisson, and D. Mounier, "Twin-Sensitivity Measurement by Spatial Multiplexing of Digitally Recorded Holograms, ” Appl. Opti. 42, 42: 1947(2003).

19. K. Kokkonen, M. Kaivola. "Scanning heterodyne laser interferometer for phase- sensitive absolute-amplitude measurements of surface vibrations," Appl.Phy.Letters 92, 063502(2008).

20. X. Dai, M. Wang, Y. Zhao and J. Zhou, "Self-mixing interference in fiber ring laser and its application for vibration measurement", Opt. Express 17(19), 16543-16548(2009) .

21. C. Hsu, Y. Sung, Z. Lin and M. Kao, "Prototype of a compact displacement sensor with a holographic diffraction grating”, Optics \& Laser Technology 48, 200-205 (2013).

22. K. Kageyama, I. Kimpara, T. Suzuki, I. Ohsawa, H. Murayama, K. Ito, "Smart marine structures: an approach to the monitoring of ship structures with fiber-optic sensors", Smart Mater. Struct. 7, 472-478 (1998)

23. M. Johansmann, G. Siegmund, M. Pineda, "Targeting the limits of laser Doppler vibrometry", in: Proceedings of the IDEMA 2005, Tokyo, Japan, 1-12 (2005).

24. S. Witte, M. Bclayon, E. J. G. Peterman, R. F.G. Toonen, H.D. Mansvelder, M. L. Groot, "Single-shot two-dimensional full-range optical coherence tomography achieved by dispersion control," Opti. Express 17(14), 11335-11349 (2009).

25. A.F. Fercher, W. Drexler, CK Hitzenberger, T. Lasse, "Optical coherence tomographyprinciples and applications," Report on Progress in Physics 66, 239-303(2003).

26. L. Chen, J. A. Zeitler, Y. Dong, Y. Shen, "Nondestructive evaluation of Polymer Coating Structures on Pharmaceutical Pellets using Full Field Optical Coherence Tomography," J. Pharm. Sci.103, 103(2014). 
27. S. Zhong, S. O. Oyadiji, "Response-only method for damage detection of beam-like structures using high accuracy frequencies with auxiliary mass spatial probing”, J. Sound Vib. 311(3), 1075(2008).

28. K. Ding, M. Xie and X. Zhang, "Phase difference correction method for phase and frequency in spectral analysis", Mechanical Systems and Signal Processing 14(5), 835 (1996) 


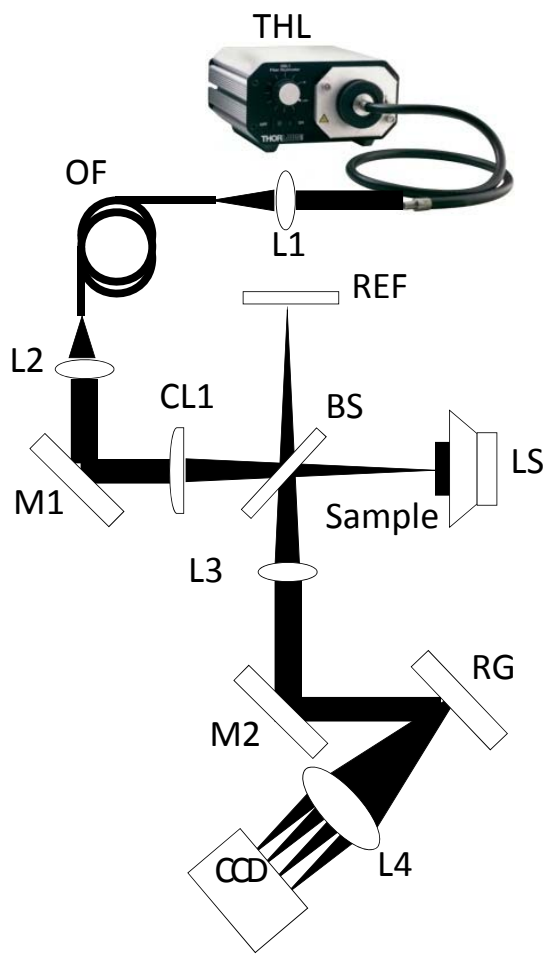

Fig. 1 The schematic diagram of a developed two-dimensional optical coherence vibration tomography (2DOCVT) system. The vibration of a sample was excited by a loudspeaker. Full spectral interferogram was recorded by a high-speed CCD camera. THL: Tungsten Halogen Lamp (OSL1-EC, Thorlabs); L: Lens; OF: Optical fiber; M: mirror; CL: Cylindrical lens; BS: 50/50 beam splitter; REF: Reference; LS: Loudspeaker; RG: Reflection grating; CCD: $1280 \times 1024$ pixels CCD camera (PCO.1200hs, PCO-TECH, Germany). 

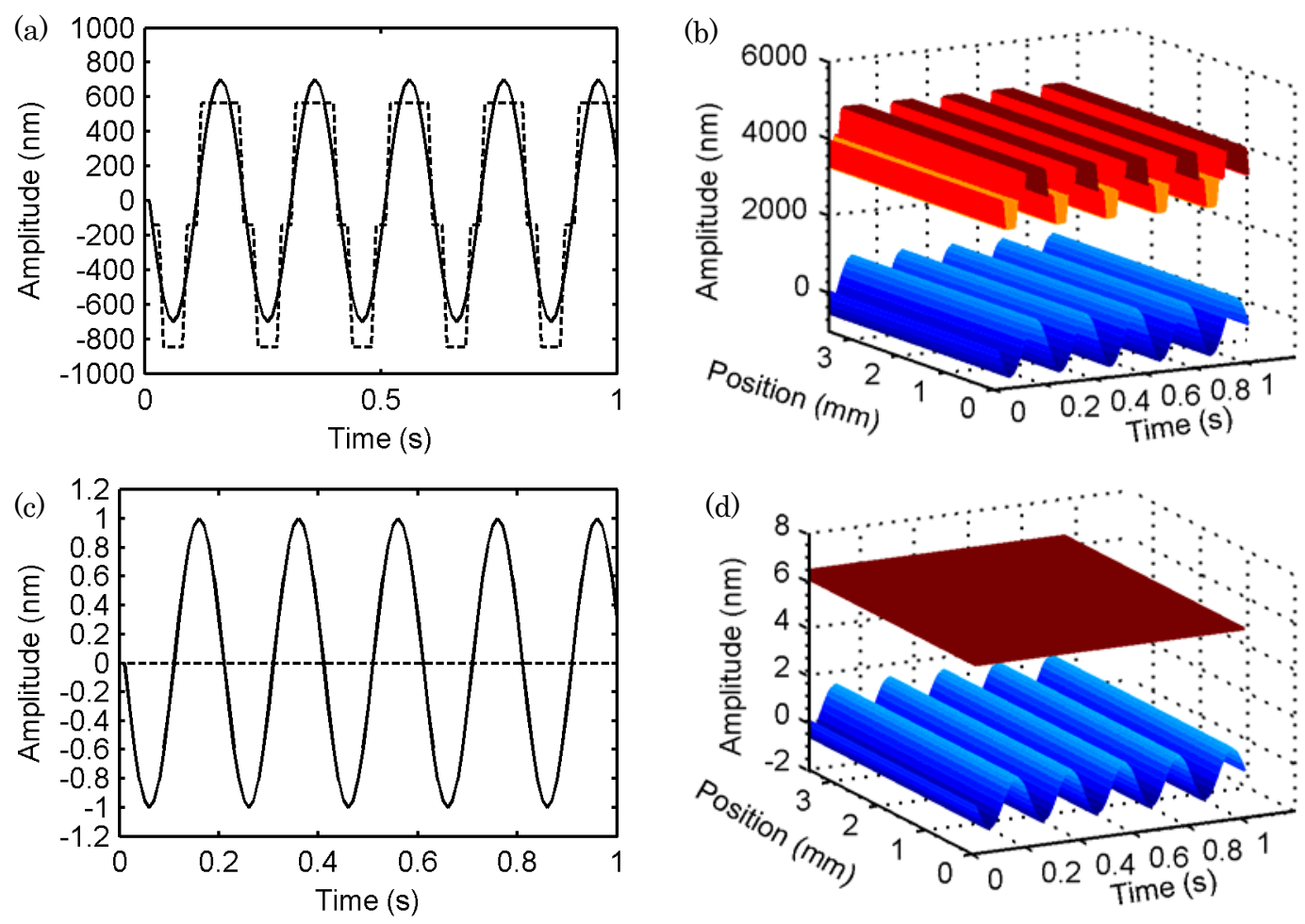

Fig.2 (a) The dashed and solid lines are the displacements of $700 \mathrm{~nm}$ sine vibration without and with spectral center correction method on simulated spectral interferogram and (b) the corresponding line vibration; (c) The dashed and solid lines are the displacements of $1 \mathrm{~nm}$ sine vibration without and with SCCM on simulated spectral interferogram and (d) the corresponding line vibration. For clarity, all the line vibrations are offset vertically. 

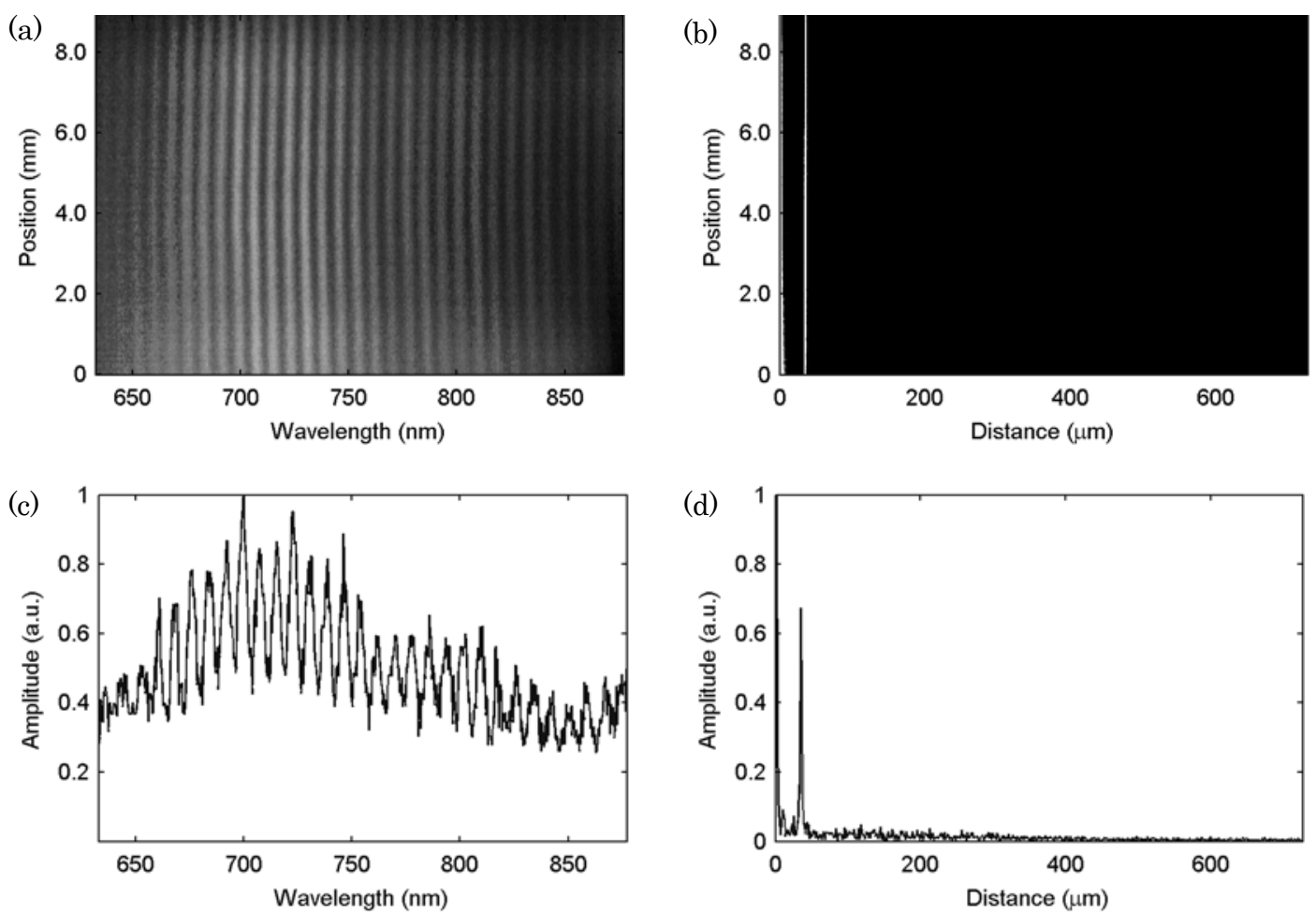

Fig. 3 (a) The interferogram of a static sample and a reference surface in the cylindrical line focus; (b) the corresponding FFT of the captured interferogram. The line at the position of $35.2 \mu \mathrm{m}$ indicated the surface position of the sample; (c) OCT spectral interferogram from No. 400 pixel of the CCD camera; (d) the corresponding FFT of OCT spectral interferogram whose peak showed the surface position of the sample. 
(a)
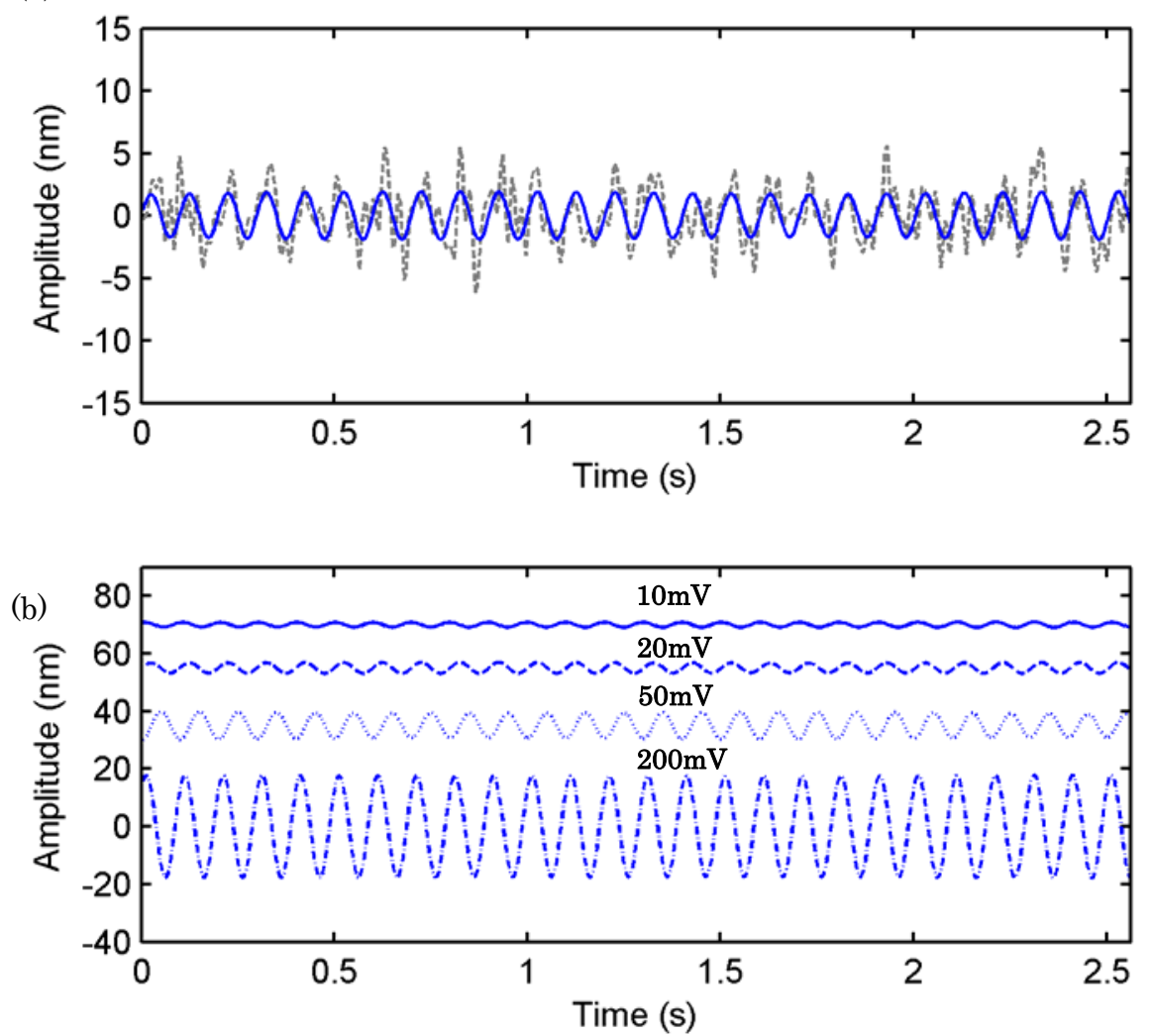

Fig.4 (a) The dashed is the noisy vibration excited by PZT actuator by $10 \mathrm{~Hz}$ sine wave with an amplitude of $20 \mathrm{mv}$ voltages whist the solid line is the filtered vibration signal with the vibration amplitude of $1.72 \mathrm{~nm}$; (b) The vibrations excited by PZT actuator by $10 \mathrm{~Hz}$ sine wave with amplitudes of $10 \mathrm{mv}, 40 \mathrm{mv}$ and $200 \mathrm{mv}$ voltages. The corresponding amplitudes for $10 \mathrm{mv}$, $40 \mathrm{mv}$ and $200 \mathrm{mv}$ voltages are respectively $0.86,4.4$ and $17.3 \mathrm{~nm}$. For clarity, all the curves are offset vertically.

(a)

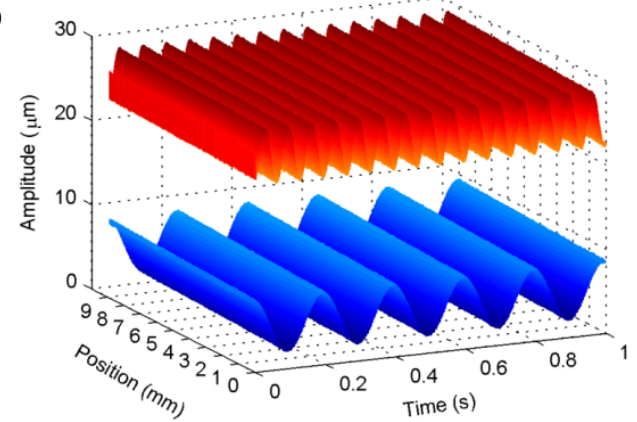

(b)

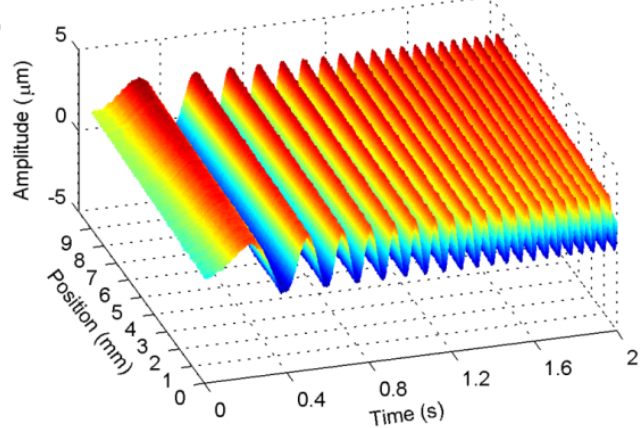

Fig.5 Line vibration measurement of a vibrating sample excited by (a) $5 \mathrm{~Hz}$ (the lower one) and $15 \mathrm{~Hz}$ (the upper one) sine signal and (b) $0-20 \mathrm{~Hz}$ sweep source using the developed 2DOCVT system. 

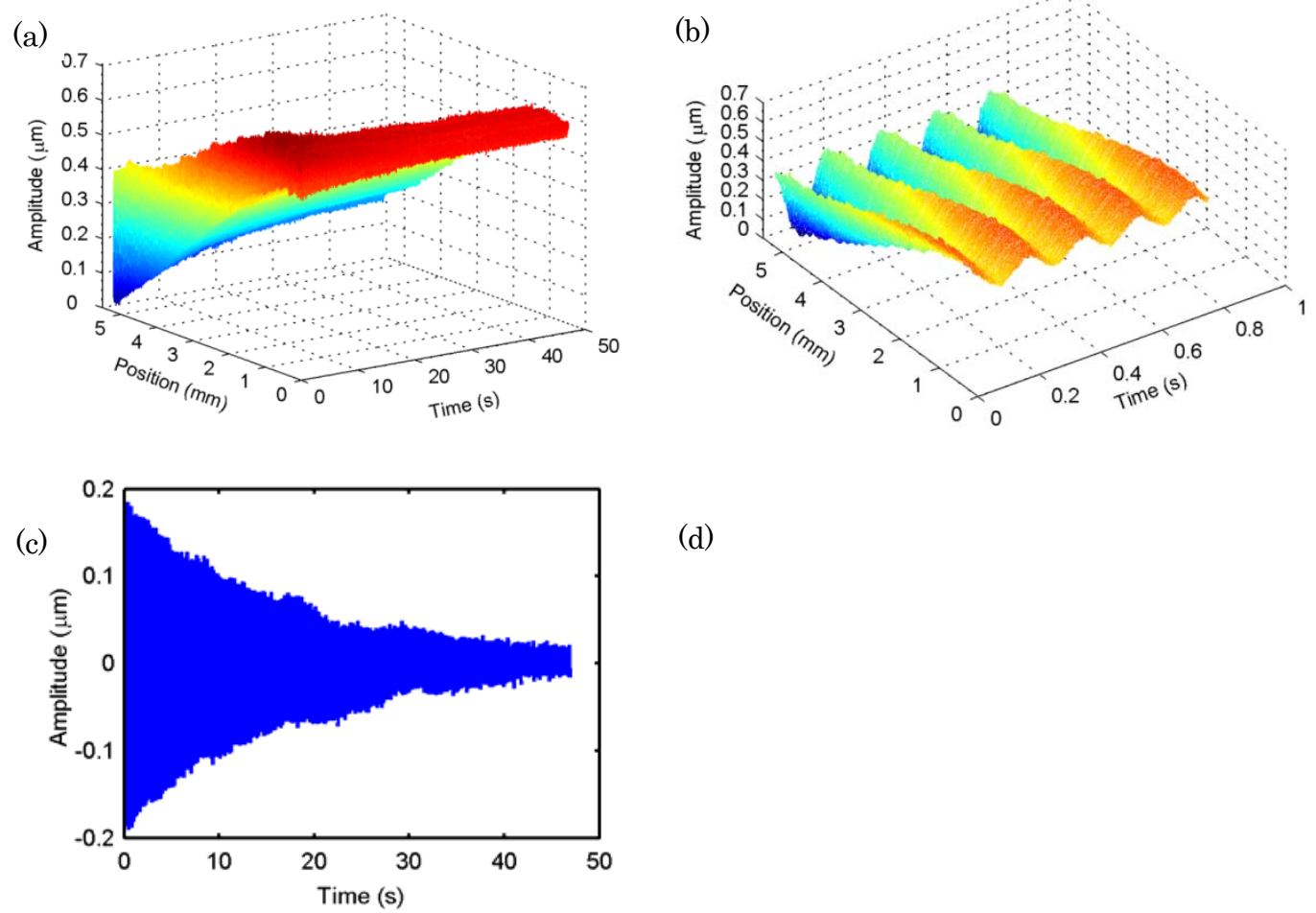

(d)

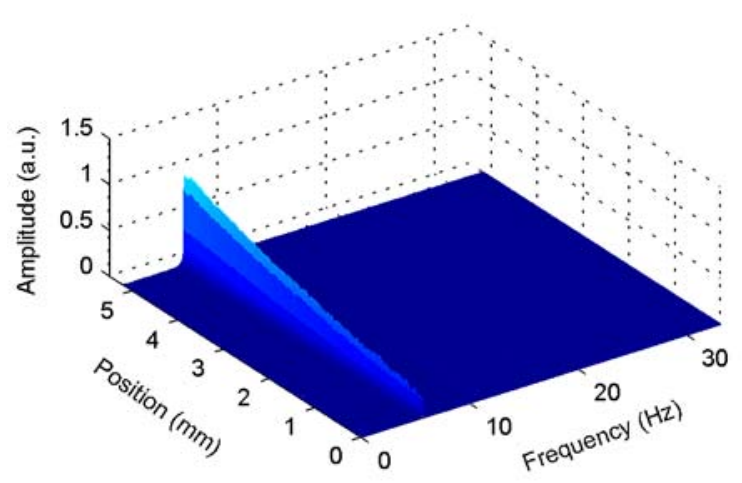

Fig. 6 (a) the line vibration of the cantilevered beam captured by 2DOCVT system during the period of $46.9 \mathrm{~s}$; (b) the zoom line vibration for the range of 0 to $0.8 \mathrm{~s}$; (c) the point vibration signal from No.270 pixel of CCD camera; (d) the FFT of the line vibration shown in (a). 